\title{
Accuracy of an ambulatory device for the diagnosis of sleep disordered breathing
}

\section{David Cunnington ${ }^{1,2}$ Himanshu Garg ${ }^{1,2}$ Harry Teichtahl ${ }^{1,2}$}

\author{
1. Department of Respiratory and Sleep Disorders Medicine, 2. Department of Medicine, Western
}

Hospital, The University of Melbourne, Australia

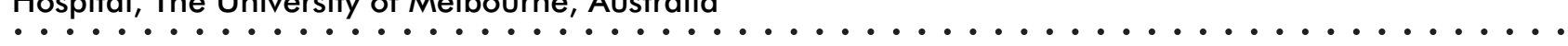

Indian J Sleep Med 2009; 4.4, 143-148

\begin{abstract}
Objective: This study aimed to evaluate the accuracy of the portable Somté ${ }^{T M}$ (Compumedics Limited, Melbourne, Australia) sleep data acquisition device against the current gold-standard of technician-attended, laboratory-based polysomnography (PSG) for the diagnosis of sleep disordered breathing (SDB).

Methods: Patients attending the sleep disorders laboratory for overnight PSG for the evaluation of suspected SDB were fitted with both the Somté device and a PSG system (Compumedics ESeries). An apnea-hypopnea index (AHI) was calculated after manual scoring of respiratory events recorded using the Somté and PSG systems.

Results: 37 patients (29 male, 8 female) participated in the study. The mean difference between the AHI estimated by the two devices showed good agreement, with the Somte device AHI being similar to that obtained by PSG. Mean difference (PSG - Somté AHI), -0.5 events per hour $(95 \% \mathrm{Cl}-4.4$ to $5.4, p=0.83)$. The Somté device was able to correctly classify the presence or absence of SDB (defined as PSG AHI $e^{\prime \prime} 15$ ) with a sensitivity of $96 \%$, specificity of $83 \%$, positive predictive value of $92 \%$ and a negative predictive value of $91 \%$.

Conclusion: The Somté device accurately detects respiratory events during sleep and can be used to diagnose SDB in patients referred for the evaluation of sleep disordered breathing.

Keywords: obstructive sleep apnea, polysomnography, diagnosis, ambulatory monitoring, physiologic monitoring
\end{abstract}

\section{Introduction}

S leep disorders are very common and sleep disordered breathing (SDB) is the most common problem in patients referred to sleep centers for assessment. The most common form of SDB is obstructive sleep apnea (OSA) which occurs in

\footnotetext{
Address for Correspondence

Dr David Cunnington, MMedSc FRACP FCCP FAASM Department of Respiratory and Sleep Disorders Medicine, Western Hospital,Gordon St, Footscray VIC 3011, Australia

Phone: +6138345 6842; Fax: +6139318 6342

email: david.cunnington@wh.org.au
}

approximately $25 \%$ of 50 year old men.(1) With obesity continuing to increase in both developed and developing countries, the prevalence of sleep disordered breathing and common co-morbid conditions such as diabetes mellitus, hypertension and cardiac failure will continue to increase. (2-5) As treatment for OSA is available, making the diagnosis of OSA accurately and in a timely fashion is important in reducing its personal and public health impact.

Access to in laboratory full polysomnography (PSG), the current gold standard for the diagnosis of OSA can be limited due to a range of factors, including geography, cost and available resources. Combined with other factors such as lack of recognition of symptoms of sleep apnea 
by patients and primary care physicians, the majority of patients with moderate to severe sleep apnea remain undiagnosed. $(6,7)$ Therefore, as new and simpler diagnostic devices become available for the detection and measurement of sleep apnea, they should be evaluated against the current gold standard of inlaboratory PSG. If their diagnostic performance is similar, the simpler devices may offer greater access for patients due to reduced cost and resources required, particularly if these devices are suitable for use in an unattended setting. Previous studies have shown that conducting sleep studies in the home is feasible and costs less than conducting studies in the hospital laboratory setting. $(8,9)$

A recent review on the use of portable devices for the diagnosis of sleep apnea has led to a recommendation by the American Academy of Sleep Medicine (AASM), American Thoracic Society (ATS) and American College of Chest Physicians (ACCP) that no type of portable monitoring device be used in an unattended setting, and only type 3 devices can be used in an attended setting to confirm the diagnosis of OSA.(10) This does not mean that such devices may not have a role for use in an unattended setting in the future; just that evidence for this has not yet been published. This study aims to add to the literature, by evaluating the SomtéTM (Compumedics, Melbourne, Australia) compared to PSG in an attended setting in patients referred for the evaluation of suspected sleep disordered breathing (SDB). If the device performs well in this setting, subsequent studies will be required to validate its use in an un-attended setting and in different patient populations.

\section{Methods}

\section{Study Design}

This study measured respiratory events during sleep with each patient wearing both Compumedics Somté and Compumedics E-Series (Compumedics Limited, Melbourne, Australia) systems simultaneously. The rate of respiratory events was then compared to assess the accuracy and diagnostic performance of the Somté device using a gold standard of technician-attended, laboratory-based (PSG). The institutional ethics committee approved the study and all study participants gave written informed consent to participate in the study.

\section{Patients}

Patients were referred for the investigation of probable OSA by sleep physicians. Those referred for investigation of non-respiratory sleep disorder, or with significant cardio-respiratory co-morbidity were excluded from the study. Eligible patients were therefore those with high pre-test probability for obstructive SDB as assessed by a sleep physician. Patients were consecutively enrolled into the study. Enrolled patients were fitted with both the Somté and E-Series devices and had simultaneous recording of sleep data using both systems over a single night in the sleep disorders laboratory at Western Hospital.

\section{Measurements}

PSG using the E-Series system was performed for all patients. Data recorded were EEG (C3-A2, C4-A1), EMG (bilateral tibialis anterior and mentalis muscle activity), EOG (left and right), ECG (modified Lead II electrocardiogram), nasal pressure, oro-nasal thermistor (Protech, Mukilteo, WA), thoracic and abdominal effort (piezo-electric bands), body position, oxygen saturation (Ohmeda 3700, Datex-Ohmeda, Madison, Wisconsin), and continuous digital video recording.

Simultaneously, data was acquired via the Somté device and recorded to a flash card for later replay and analysis. The Somté device was configured to record one channel of EEG (C3-A2 or C4-A1), ECG (modified Lead II electrocardiogram), nasal pressure, thoracic and abdominal effort (inductance plethysmography), body position, leg movements (piezo sensor) and oxygen saturation (oximeter). The Somté device allows full data disclosure, with data being reviewed using Profusion 2 software (Compumedics Limited, Melbourne, Australia)

\section{Data Analysis}

All studies were staged and scored using Profusion 2 software by the same senior registered polysomnography technician (RPSGT) who was blinded to all study details. Studies were presented to the scientist in random order.

Data from the E-Series system had sleep staging performed according to standard criteria.(11) The Somté system recorded a single channel of EEG data which was used to help identify respiratory events by looking for signs of arousal, but not used to stage sleep or determine total sleep time. Respiratory events measured 
on each system were scored according to AASM criteria.(12) Apneas were scored if there was absence of airflow for at least 10 seconds. Hypopneas were scored if there was any reduction in airflow measured by nasal pressure or oro-nasal thermistor for at least 10 seconds, together with either an arousal or oxygen desaturation of at least 3\%. In addition, hypopneas were scored without arousal or desaturation if there was a greater than $50 \%$ decrease in airflow measured by either nasal pressure or oro-nasal thermistor for at least 10 seconds. Respiratory event related arousals (RERAs) were not scored unless they met the above criteria.

The apnea-hypopnea index (AHI) for the E-Series was calculated as the total number of respiratory events occurring during sleep, divided by the total sleep time. For the Somté device, the AHI was calculated as the total number of respiratory events occurring from lights off to lights on, divided by the total time from lights off to lights on (total time available for sleep - TTA).

\section{Statistics}

The primary outcome of the study was comparison of the AHI generated from each device using paired comparison of means for normally distributed data. From pilot data in our laboratory we calculated that 35 patients would be required to detect a 10 point difference in AHI between the 2 devices, with $80 \%$ power and alpha of 0.05 . As we did not expect any patients to dropout of the study, we planned to enroll 35 patients. In addition, agreement and bias between the AHI measured by the 2 devices was assessed using Bland Altman plots. The diagnostic ability of the Somté device to correctly classify patients into having or not having SDB was also assessed. The AHI derived from the E-Series device was defined as the gold standard. Receiver operator characteristic (ROC) analysis was used to determine the Somté AHI with the best diagnostic performance to correctly classify patients as having versus not having sleep apnea, defined as an ESeries of $\mathrm{AHI} \geq 15$. Statistical analysis was performed using STATA (Version 8.0, Stata Corporation, College Station, Texas).

\section{Results}

Thirty seven patients participated in the study (29 male, 8 female). The patients had a mean (range) age of 42 (25-68), and BMI of $32 \mathrm{~kg} / \mathrm{m}^{2}$ (19-54). They had sleep propensity at the upper limit of normal with a mean Epworth Sleepiness Score of 10.2 (range 0-22).
Despite being fitted with 2 sleep monitoring devices, most patients slept for a reasonable period of time with a mean TST of 347 minutes (SD 68, range 151-461) as measured by E-series PSG. The group had a high prevalence of SDB that was relatively severe with a mean AHI (measured by E-series PSG and calculated as the total number of events divided by TST) of 34.1 (SD 29.3, range 4.8-128). Of the 37 patients, 12 (32\%) had an $\mathrm{AHI}<15,10(27 \%)$ and $\mathrm{AHI}$ of $\geq 15$ and $<30$, and 15 $(41 \%)$ an $\mathrm{AHI} \geq 30$.

The Somté device AHI, calculated as the total number of respiratory events divided by TTA, was similar to the E-Series AHI with the mean difference (E-Series AHI minus Somté AHI) between the AHIs being 0.51 events per hour $(95 \% \mathrm{CI} 4.4$ to $5.4, \mathrm{p}=0.83)$. Bland-Altman plots showed good agreement between the two devices. (Figure 1a) There were two outliers at high AHI where the Somté device significantly underestimated the AHI. At such high AHIs this would not alter clinical

Panel a:

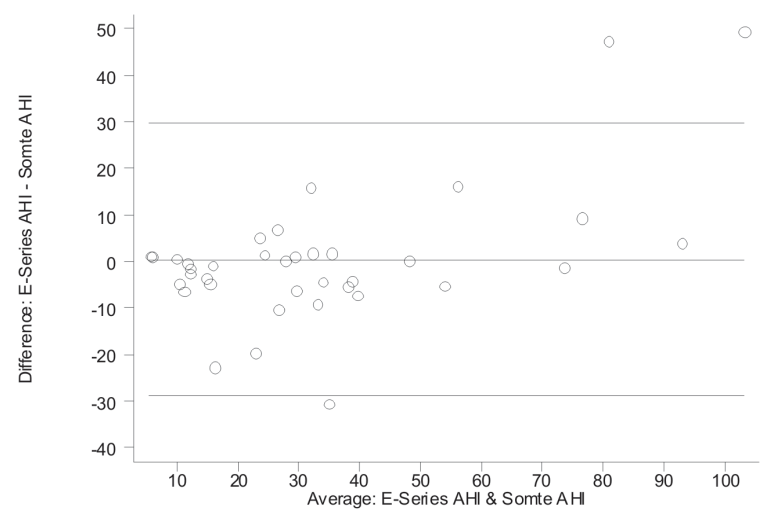

Panel b:

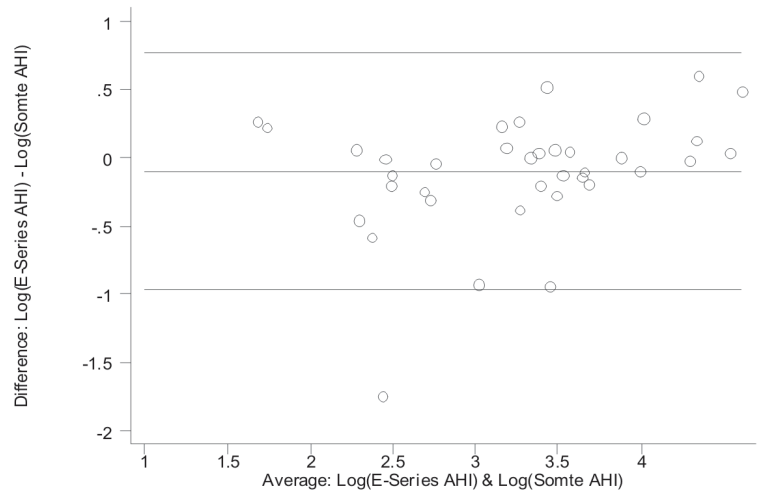

Figure 1: Bland-Altman plot of mean difference with $95 \%$ limits of agreement between AHI measured by Somté and E-Series devices. Raw values shown on panel (a), and log transfored values on panel (b) reducing the effect of increasing variability as AHI increases.

Indian Journal of Sleep Medicine (IJSM), Vol. 4, No. 4, 2009 
management, and when the data was log transformed to account for increasing variability as AHI increased, there was no evidence of systematic bias. (Figure 1b)

The Somté device accurately classified the study population into having or not having SDB (defined as an AHI of 15 measured by PSG), with the best performance seen using a cut-off of an AHI of 21 measured by Somté device. Using this cut-off, the sensitivity is $96 \%$, specificity $83 \%$, positive predictive value $92 \%$, negative predictive value $91 \%$, and the positive and negative likelihood ratios are 5.76 and 0.048 respectively. These positive and negative likelihood ratios are all in the range that are clinically useful in ruling in and out disorders across a range of AHIs. $(13,14)$ From Table 1, it can be seen that the Somté device also performed well, if the threshold used to define the presence of sleep apnea was a PSG measured AHI of $\geq 20$ or $\geq 30$, but less well at a lower AHI of $\geq 10$.

Table 1: Diagnostic performance of Somté device

\begin{tabular}{|l|l|l|l|l|l|l|l|l|}
\hline & AUC & $\begin{array}{l}\text { Somté } \\
\text { AHI }\end{array}$ & Sens & Spec & PPV & NPV & +LR & -LR \\
\hline AHI 10 & 0.894 & 12 & 96.7 & 40.0 & 91.2 & 65.4 & 1.61 & 0.078 \\
\hline AHI 15 & 0.947 & 21 & 96.0 & 83.3 & 92.3 & 90.9 & 5.76 & 0.048 \\
\hline AHI 20 & 0.910 & 23 & 95.7 & 78.6 & 88.0 & 91.8 & 4.46 & 0.055 \\
\hline AHI 30 & 0.933 & 35 & 86.7 & 90.9 & 86.7 & 90.9 & 9.53 & 0.147 \\
\hline
\end{tabular}

$A U C=$ Area under curve from ROC analysis, Sens $=$ sensitivity, Spec = specificity, $P P V=$ positive predictive value, $N P V=$ negative predictive value, $+L R=$ positive likelihood ratio, $-L R=$ negative likelihood ratio

\section{Discussion}

This study has demonstrated that the Somté device accurately detects respiratory events during sleep, and that an AHI derived from the Somté, calculated as the total number of respiratory events divided by TTA, can be used to diagnose SDB in patients with high pre-test probability of SDB.

When assessing the performance of any new diagnostic device, it is important not only to look at sensitivity, specificity, but also the positive and negative likelihood ratios. These measures are more easily translated into clinical practice and not subject to change depending upon the prevalence of the target disorder in the tested population in the way that sensitivity, specificity and the predictive values are.(15) Presenting the data in this form is clinically relevant as it allows the determination of post-test odds of SDB using Bayes's theorem and a nomogram or simple calculation, once the pre-test odds of SDB have been determined during clinical assessment. $(15,16)$ The positive and negative likelihood ratios of 5.76 and 0.048 respectively to detect an AHI of e" 15 , are clinically useful and show that the Somté device was able to accurately diagnose SDB. These results are comparable to other level 3 devices used in an attended setting for the diagnosis of SDB.(14, 17-19)

In our analysis, we evaluated the performance of the Somté device over a range of AHIs. The recommended level of an AHI of 5 or greater to diagnose SDB is based on epidemiological data and PSG using thermistors as the measure of airflow.(12) In our study nasal pressure as well as oronasal thermistor was used to measure airflow for the attended in laboratory standard PSG. Nasal pressure has been shown to be a more sensitive measure of airflow than thermistor, with Norman et al showing that thermistor only detected up to $55.7 \%$ of apneas and hypopneas detected by nasal pressure.(20) We have shown that when nasal pressure and thermistor are used in combination, more respiratory events are scored than with either signal used alone, and nasal pressure is more sensitive at low AHIs.(21) Using a combination of thermistor and nasal pressure we have also shown a mean \pm SD AHI in a non-obese control group of $9.4 \pm 9.1$, suggesting that using a cutoff of 15 to define the presence or absence of SDB may be more appropriate when nasal pressure is used to measure airflow.(22) This is supported by Hosslet et al, who found that an AHI cut-off of 15 best predicted excessive daytime sleepiness using similar respiratory event scoring criteria as used in this study.(23) Controversy surrounding the true cut-off between normal and abnormal led us to report the diagnostic performance of the Somté device over a range of thresholds for the diagnosis of SDB. This analysis showed that the Somté device performed best using an AHI of $\geq 15$ as the definition of SDB, but also performed well at higher thresholds of $\geq 20$ or $\geq 30$. The Somté device was sensitive, but not specific when a threshold of AHIe" 10 was used, in part due to the high prevalence of SDB in the patient population in this study, with only 5 of 37 (14\%), having an AHI of $<10$.

We acknowledge there are disadvantages with limited PSG. For many patients full PSG is required to determine the exact relationship between sleep stage, arousal, leg movements and respiratory changes. However, in every sleep disorders center there is a subset of patients where the clinical question is one of the 
diagnosis or exclusion of significant SDB. This study was performed on such a population, with a high pretest probability of obstructive SDB. In this population the Somté device compared very well with conventional PSG with positive and negative likelihood ratios that are helpful in clinical practice.(13) However, these results may not apply to different populations, so caution should be used in generalising these results to a broader population, such as screening a low risk population, where the diagnostic performance may not be as accurate.

Although this study did not assess the use of the Somté device in an unattended setting, we believe that similar diagnostic accuracy could be achieved in such a setting. We expect no difference in the recording of data, with the only difference in an un-attended setting being potential data loss from disconnected leads or poor signals.

As part of the diagnostic armamentarium of a sleep disorders service, a limited PSG device can be useful to increase accessibility to diagnostic and treatment of sleep disorders. We feel that such a device is best used where a qualified sleep specialist carefully selects patients and the facility exists for traditional technician-attended, laboratory-based PSG whenever required. This allows patients to be triaged through a range of diagnostic tests for SDB depending upon the presence of cardiac and respiratory co-morbidities, features of non-respiratory sleep disorders and pre-test probability of sleep disordered breathing. In such a clinical setting, we have shown that the Somté device accurately measures SDB compared to technician-attended, laboratory-based PSG, and remains accurate over a range of $\mathrm{AHI}$ values, and as such can be used to diagnose SDB in patients with a high clinical pre-test probability of SDB.

\section{Acknowledgements}

This study was supported by Compumedics Limited (Melbourne, Australia) and Respiratory Research Fund, Western Hospital.

\section{References}

1. Young T, Palta M, Dempsey J, Skatrud J, Weber S, Badr S. The occurrence of sleep-disordered breathing among middle-aged adults. N Engl J Med. 1993;328(17):1230-5.

2. Reddy EV, Kadhiravan T, Mishra HK, Sreenivas V, Handa KK, Sinha S, et al. Prevalence and risk factors of obstructive sleep apnea among middle-aged urban Indians: a community-based study. Sleep Med. 2009 Sep;10(8):913-8.

3. Sharma SK, Kumpawat S, Banga A, Goel A. Prevalence and risk factors of obstructive sleep apnea syndrome in a population of Delhi, India. Chest. 2006 Jul;130(1):149-56.

4. The burden of overweight and obesity in the Asia-Pacific region. Obes Rev. 2007 May;8(3):191-6.

5. Ogden CL, Carroll MD, Curtin LR, McDowell MA, Tabak CJ, Flegal KM. Prevalence of overweight and obesity in the United States, 1999-2004. Jama. 2006 Apr 5;295(13):1549-55.

6. Young T, Evans L, Finn L, Palta M. Estimation of the clinically diagnosed proportion of sleep apnea syndrome in middleaged men and women. Sleep. 1997 Sep;20(9):705-6.

7. Flemons WW, Douglas NJ, Kuna ST, Rodenstein DO, Wheatley J. Access to diagnosis and treatment of patients with suspected sleep apnea. Am J Respir Crit Care Med. 2004 Mar 15;169(6):668-72

8. Parra O, Garcia-Esclasans N, Montserrat JM, Garcia Eroles L, Ruiz J, Lopez JA, et al. Should patients with sleep apnoea/ hypopnoea syndrome be diagnosed and managed on the basis of home sleep studies? Eur Respir J. 1997; 10(8):1720-4.

9. Kapur VK, Rapoport DM, Sanders MH, Enright P, Hill J, lber $C$, et al. Rates of sensor loss in unattended home polysomnography: the influence of age, gender, obesity, and sleep-disordered breathing. Sleep. 2000;23(5):682-8.

10. Chesson AL, Jr., Berry RB, Pack A. Practice parameters for the use of portable monitoring devices in the investigation of suspected obstructive sleep apnea in adults. Sleep. 2003 Nov 1;26(7):907-13.

11. Rechtschaffen A, Kales A. A Manual of Standardized Terminology and Scoring System for Sleep Stages in Human Subjects. Washington, DC: National Institutes of Health; 1968.

12. Sleep-related breathing disorders in adults: recommendations for syndrome definition and measurement techniques in clinical research. The Report of an American Academy of Sleep Medicine Task Force. Sleep. 1999 Aug 1;22(5):667-89.

13. Simel D, Feussner J, DeLong E, Matchar D. Intermediate, indeterminate, and uninterpretable diagnostic results. Med Decis Making. 1987;7(2):107-14.

14. Flemons WW, Littner MR, Rowley JA, Gay P, Anderson WM, Hudgel DW, et al. Home diagnosis of sleep apnea: a systematic review of the literature. An evidence review cosponsored by the American Academy of Sleep Medicine, the American College of Chest Physicians, and the American Thoracic Society. Chest. 2003 Oct;124(4):1543-79.

15. Sackett DL, Haynes RB, Guyatt G, Tugwell P. The interpretation of diagnostic data. Clinical Epidemiology: a basic science for clinical medicine. 2nd edition ed. Boston: Little, Brown and Company; 1991. p. 69-152.

16. Fagan TJ. Letter: Nomogram for Bayes theorem. N Engl J Med. 1975 Jul 31;293(5):257.

17. Zucconi M, Ferini-Strambi L, Castronovo V, Oldani A, 
Smirne S. An unattended device for sleep-related breathing disorders: validation study in suspected obstructive sleep apnoea syndrome. Eur Respir J. 1996 Jun;9(6):1251-6.

18. Verse T, Pirsig W, Junge-Hulsing B, Kroker B. Validation of the POLY-MESAM seven-channel ambulatory recording unit. Chest. 2000;117(6):1613-8.

19. Emsellem HA, Corson WA, Rappaport BA, Hackett S, Smith LG, Hausfeld JN. Verification of sleep apnea using a portable sleep apnea screening device. South Med J. 1990 Jul;83(7):748-52.

20. Norman RG, Ahmed MM, Walsleben JA, Rapoport DM. Detection of repsiratory events during NPSG: nasal cannula/ pressure sensor versus thermistor. Sleep. $1997 ; 20(12): 1175-84$
21. Teichtahl H, Cunnington D, Cherry G, Wang D. Scoring polysomnography respiratory events: the utility of nasal pressure and oro-nasal thermal sensor recordings. Sleep Med. 2003 Sep;4(5):419-25.

22. Wang D, Teichtahl H, Drummer O, Goodman C, Cherry G, Cunnington D, et al. Central sleep apnea in stable methadone maintenance treatment patients. Chest. 2005 Sep; $128(3): 1348-56$.

23. Hosselet J, Ayappa I, Norman RG, Krieger AC, Rapoport DM. Classification of sleep-disordered breathing. Am J Respir Crit Care Med. 2001 Feb;163(2):398-405. 